This item was submitted to Loughborough's Research Repository by the author.

Items in Figshare are protected by copyright, with all rights reserved, unless otherwise indicated.

\title{
Embedded eigenvalues of generalized Schrodinger operators
}

PLEASE CITE THE PUBLISHED VERSION

https://doi.org/10.4171/JST/298

PUBLISHER

European Mathematical Society

VERSION

AM (Accepted Manuscript)

PUBLISHER STATEMENT

This paper was accepted for publication in the journal Journal of Spectral Theory and the definitive published version is available at https://doi.org/10.4171/JST/298.

\section{LICENCE}

CC BY-NC-ND 4.0

\section{REPOSITORY RECORD}

Cuenin, Jean-Claude. 2020. "Embedded Eigenvalues of Generalized Schrodinger Operators”. Loughborough University. https://hdl.handle.net/2134/12928523.v1. 


\title{
EMBEDDED EIGENVALUES OF GENERALIZED SCHRÖDINGER OPERATORS
}

\author{
JEAN-CLAUDE CUENIN
}

\begin{abstract}
We provide examples of operators $T(D)+V$ in $L^{2}\left(\mathbb{R}^{d}\right)$ with decaying potentials that have embedded eigenvalues. The decay of the potential depends on the curvature of the (Fermi) surfaces of constant kinetic energy $T$. We make the connection to counterexamples in Fourier restriction theory.
\end{abstract}

\section{Introduction}

The question of whether the Schrödinger operator

$$
-\Delta+V
$$

with a decaying potential $V$ can have eigenvalues embedded in the continuous spectrum is notoriously difficult. On physical grounds, one has good reasons to expect that the potential cannot prevent a particle from escaping to spatial infinity, due to quantum mechanical tunneling. This argument would lead one to believe that embedded eigenvalues cannot occur. It came as a big surprise in the early days of quantum mechanics when Wigner and von Neumann [28] (see also [21, Section XII.13] for a corrected proof) found an example of a bounded potential that decays like $1 /|x|$ at infinity such that the operator (1) has an embedded eigenvalue $\lambda=1$. The crucial feature of the potential is its delicate oscillatory pattern, which causes waves to be reflected coherently. On the other hand, Kato [15] proved that if $V$ is bounded and $V(x)=o(1 /|x|)$ as $|x| \rightarrow \infty$, then there are no embedded eigenvalues (see [1] and [22] for the case of long-range perturbations, which we will not discuss here.)

A different example leading to an embedded eigenvalue for (1) was constructed by Ionescu and Jerison [11]. The Ionescu-Jerison potential is bounded, decays like $1 /|x|$ in one coordinate direction and like $1 /|x|^{2}$ in the others. While the Wignervon Neumann construction is reduced to a one-dimensional problem by spherical symmetry, the Ionescu-Jerison construction is inherently multi-dimensional. Note that the Wigner-von Neumann potential is in $L^{q}\left(\mathbb{R}^{d}\right)$ for any $q>d$, while the Ionescu-Jerison potential is in $L^{q}\left(\mathbb{R}^{d}\right)$ for any $q>(d+1) / 2$. Koch and Tataru [18] proved the absence of embedded eigenvalues for (1) for a large class of potentials including $L^{(d+1) / 2}\left(\mathbb{R}^{d}\right)$. This is sharp in view of the Ionescu-Jerison example. Furthermore, since the eigenfunction is not rapidly decaying (see (7)), the same

1991 Mathematics Subject Classification. Primary 35J10; Secondary 42B37, 35S05.

Key words and phrases. Embedded eigenvalues, Schrödinger operator, pseudodifferential operators, Dirac operator, Fourier restriction. 
example shows optimality of the Agmon-Kato-Kuroda theorem, as proved by Ionescu and Schlag [12] in the setting of $L^{q}$ potentials.

Recently, Frank and Simon [9] adapted and simplified the two examples to show that embedded eigenvalues may occur for potentials with arbitrary small $L^{q}$ norms, More precisely, they proved the following.

(A) There exists a sequence of potentials $V_{n} \in L^{q}\left(\mathbb{R}^{d}\right)$ for any $q>(d+1) / 2$ such that $-\Delta+V_{n}$ has eigenvalue $\lambda=1$ and $\lim _{n \rightarrow \infty}\left\|V_{n}\right\|_{L^{q}\left(\mathbb{R}^{d}\right)}=0$.

(B) There exists a sequence of radial potentials $V_{n} \in L^{q}\left(\mathbb{R}^{d}\right)$ for any $q>d$ such that $-\Delta+V_{n}$ has eigenvalue $\lambda=1$ and $\lim _{n \rightarrow \infty}\left\|V_{n}\right\|_{L^{q}\left(\mathbb{R}^{d}\right)}=0$.

The potentials can be chosen real-valued, so that (1) is self-adjoint. Conversely, they prove that even for complex-valued potentials $V \in L^{q}\left(\mathbb{R}^{d}\right)$, with $d / 2 \leq q \leq$ $(d+1) / 2$ in the general case and $d / 2 \leq q \leq d$ in the radial case (in both cases $q \neq d / 2$ if $d=2)$, every eigenvalue $\lambda \in \mathbb{C}$ of (1) satisfies

$$
|\lambda|^{1-d /(2 q)} \leq C_{d, q}\|V\|_{L^{q}\left(\mathbb{R}^{d}\right)} .
$$

Since the exponent of $|\lambda|$ is nonnegative, (2) implies the absence of eigenvalues in $(0, \infty)$ for potentials with small $L^{q}$ norm. The result in the general case was proved before by Frank [8] for non-embedded eigenvalues, i.e. $\lambda \in \mathbb{C} \backslash[0, \infty)$. In view of (A)-(B), the assumption on the upper bound for $q$ are optimal. Counterexamples for $q<d / 2$ are of a very different nature and we will not consider them here. We just mention [16] and [17] where examples of unbounded, compactly supported potentials $V \in L^{q}\left(\mathbb{R}^{d}\right)$ (with $q<d / 2$ ) were constructed such that (1) has eigenvalue $\lambda=1$.

There is an interesting connection between the above two examples (Wignervon Neumann and Ionescu-Jerison) and counterexamples in the Fourier restriction problem. In its dual form, the latter asks for which exponents $p, q \in[1, \infty]$ the inequality

$$
\|\widehat{g \mathrm{~d} \sigma}\|_{L^{p^{\prime}}\left(\mathbb{R}^{d}\right)} \leq C\|g\|_{L^{q^{\prime}\left(S^{d-1}\right)}}
$$

holds for any $g \in L^{q^{\prime}}\left(S^{d-1}\right)$. Here, $\mathrm{d} \sigma$ is the canonically induced surface measure on $S^{d-1}$. Necessary conditions for (3) to hold are

$$
1 \leq p<\frac{2 d}{d+1}, \quad \frac{1}{q} \geq \frac{d+1}{d-1} \frac{1}{p^{\prime}}
$$

see e.g. [24]. Whether these conditions are also sufficient is one of the major open problems in harmonic analysis, known as the restriction conjecture. For $q=2$ it is known to be true, i.e. (3) holds for

$$
1 \leq p \leq \frac{2(d+1)}{d+3} .
$$

This is the content of the Stein-Tomas theorem (see [24, Proposition IX.2.1]). If one restricts the class of $g$ in (3) to radial functions, then the first condition in (4) is in fact sufficient (see [25, Chapter 8, Proposition 5.1]). The necessity follows 
from the fact that

$$
\begin{aligned}
\widehat{\mathrm{d} \sigma}(x) & =2 \pi|x|^{-\frac{d-2}{2}} J_{\frac{d-2}{2}}(|x|) \\
& =2 \sqrt{2 \pi}|x|^{-\frac{d-1}{2}} \cos \left(|x|-\frac{\pi(d-1)}{4}\right)+\mathcal{O}\left(|x|^{-\frac{d+1}{2}}\right)
\end{aligned}
$$

as $|x| \rightarrow \infty$ and by taking $g=1$ in (3). In the nonradial case, the necessity of the second condition in (4) follows from the so-called Knapp example (see e.g. [10] or [20] for a textbook presentation). The connection to the problem of embedded eigenvalues is twofold. First, the numerology is such that

$$
\begin{aligned}
& \mathcal{B}\left(L^{p}\left(\mathbb{R}^{d}\right), L^{p^{\prime}}\left(\mathbb{R}^{d}\right)\right)=L^{d}\left(\mathbb{R}^{d}\right) \quad \text { for } \quad p=\frac{2 d}{d+1}, \\
& \mathcal{B}\left(L^{p}\left(\mathbb{R}^{d}\right), L^{p^{\prime}}\left(\mathbb{R}^{d}\right)\right)=L^{\frac{d+1}{2}}\left(\mathbb{R}^{d}\right) \quad \text { for } \quad p=\frac{2(d+1)}{d+3} .
\end{aligned}
$$

Here $\mathcal{B}\left(L^{p}\left(\mathbb{R}^{d}\right), L^{p^{\prime}}\left(\mathbb{R}^{d}\right)\right)$ denotes the space of bounded operators from $L^{p}$ to $L^{p^{\prime}}$. On the left, we have precisely the endpoints in (4), (5). On the right, we have the endpoints for $q$ in (A), (B). Second, the eigenfunction used in [9] for the Wigner-von Neumann example is $u(x)=\widehat{\mathrm{d} \sigma}(x) w(x)$, where $w$ is a positive radial function. This is the first (and quite obvious) connection. The eigenfunction in the simplest version (the resulting potential is complex-valued in this case) of the Ionescu-Jerison example [11] has the form

$$
u(x)=\mathrm{e}^{\mathrm{i} x_{d}}\left(1+\left|x^{\prime}\right|^{4}+\left|x_{d}\right|^{2}\right)^{-N / 2},
$$

where $x=\left(x^{\prime}, x_{d}\right) \in \mathbb{R}^{d-1} \times \mathbb{R}$ and $N>(d+1) / 4$ (this ensures that $u \in L^{2}\left(\mathbb{R}^{d}\right)$ ). The parabolic scaling is characteristic for Knapp type examples. In fact, it is not difficult to show that $u$ is a superposition of infinitely many Knapp examples (see Section 3).

The significance of the unit sphere in the above discussion is that it coincides with the Fermi surface for the dispersion relation $T(\xi)=|\xi|^{2}$ at energy $\lambda=1$. For arbitrary smooth kinetic energies (dispersion relations) $T$, the Fermi surface at energy $\lambda$ (assumed to be a regular value of $T$ ) is given by

$$
M_{\lambda}=\left\{\xi \in \mathbb{R}^{d}: T(\xi)=\lambda\right\} .
$$

If $M_{\lambda}$ is compact and has everywhere non-vanishing Gussian curvature, the conclusion of the Stein-Tomas theorem still holds, i.e. (3) with $q=2$ and $p$ as in (5) is true. Moreover, analogues of the bound (2) were proved in [5] for some special choices of the kinetic energy.

The aim of this note is to construct examples of embedded eigenvalues for generalized Schrödinger operators, i.e. operators of the form

$$
T(D)+V .
$$

Here $V$ is a decaying potential and the translation-invariant kinetic energy operator $T(D)$ is a Fourier multiplier acting on Schwartz functions $f$ as

$$
(T(D) f)(x):=(2 \pi)^{-d} \int_{\mathbb{R}^{d}} \mathrm{e}^{\mathrm{i} x \cdot \xi} T(\xi) \widehat{f}(\xi) \mathrm{d} \xi .
$$


The convention for the Fourier transformation used here is

$$
\widehat{f}(\xi):=\int \mathrm{e}^{-\mathrm{i} x \cdot \xi} f(x) \mathrm{d} x, \quad f \in L^{1}\left(\mathbb{R}^{d}\right) .
$$

All integrals will be over $\mathbb{R}^{d}$ unless otherwise indicated and $D_{j}=-\mathrm{i} \partial_{j}$ for all $j=1, \ldots, d$.

In the following we generalize the statements (A) and (B) above to operators of the form (10). The main examples involve complex-valued potentials (see Theorems 1.1-1.2) but under additional assumptions on $T$ the potential $V$ can be real-valued (see Theorem 1.3 and Section 4 ). Since the Fourier transform intertwines complex conjugation with the parity operator $(P \psi)(x)=\psi(-x)$, the symbol $T$ should at least be time-reversal symmetric, i.e. $T(\xi)=T(-\xi)$ (see Lemma 4.1). We expect that this condition is sufficient to get real-valued $V$, but currently we can only prove this under further assumptions on $T$. As a special case we consider the "Chandrasekhar-Herbst operator" $\sqrt{-\Delta+1}-1$ (see Theorem 1.4). This generalizes a recent result of Lorinczi and Sasaki [19] to nonradial potentials.

Our final example shows that the construction of the counterexamples also works for some matrix-valued differential operators. Here we consider the Dirac operator (with or without mass). A straightforward adaptation of the previous arguments yields an anti-hermitian potential (see Theorem 1.5). It would be interesting to produce an example with a hermitian potential. For this one would probably have to exploit the matrix structure of the Dirac operator more fully, but we do not pursue this here. For results on the absence of embedded eigenvalues of the Dirac operato we refer e.g. to $[2,27,3,4]$ and to $[14,7]$ and references therein for a discussion of earlier results.

The following are the main results of this note.

Theorem 1.1 (Complex-valued potentials). Let $T: \mathbb{R}^{d} \rightarrow \mathbb{C}$ be smooth and polynomially bounded, and let $\lambda \in \mathbb{C}$ be a regular value. Then there exists a sequence of smooth potentials $V_{n}: \mathbb{R}^{d} \rightarrow \mathbb{C}, n \in \mathbb{N}$, satisfying (possibly after rotating coordinates)

$$
\left|V_{n}(x)\right| \lesssim\left(n+\left|x_{1}\right|^{2}+\ldots+\left|x_{d-1}\right|^{2}+\left|x_{d}\right|\right)^{-1}
$$

and such that $\lambda$ is an eigenvalue of $T(D)+V_{n}(x)$ in $L^{2}\left(\mathbb{R}^{d}\right)$ for every $n \in \mathbb{N}$. In particular, for any $q>(d+1) / 2$, we have that $\lim _{n \rightarrow \infty}\left\|V_{n}\right\|_{L^{q}}=0$.

Theorem 1.2 (Complex-valued potentials II). Let $T: \mathbb{R}^{d} \rightarrow \mathbb{R}$ be smooth and polynomially bounded, and let $\lambda \in \mathbb{R}$ be a regular value (hence $M_{\lambda}$ is a smooth hypersurface). Assume that $M_{\lambda}$ has $k<d-1$ non-vanishing principal curvatures at some point. Then there exists a sequence of smooth potentials $V_{n}: \mathbb{R}^{d} \rightarrow \mathbb{C}$, $n \in \mathbb{N}$, satisfying (possibly after rotating coordinates)

$$
\left|V_{n}(x)\right| \lesssim\left(n+\left|x_{1}\right|^{2}+\ldots+\left|x_{k}\right|^{2}+\left|x_{k+1}\right|^{3}+\ldots+\left|x_{d-1}\right|^{3}+\left|x_{d}\right|\right)^{-1}
$$

and such that $\lambda$ is an eigenvalue of $T(D)+V_{n}(x)$ in $L^{2}\left(\mathbb{R}^{d}\right)$, for all $n \in \mathbb{N}$. In particular, for any $q>(k+2) / 2+(d-1-k) / 3$, we have that $\lim _{n \rightarrow \infty}\left\|V_{n}\right\|_{L^{q}}=0$.

Theorem 1.3 (Real-valued, radial potentials). Let $T: \mathbb{R}^{d} \rightarrow \mathbb{R}$ be a nonconstant, radially symmetric polynomial, and let $\lambda \in \mathbb{R}$ be a regular value. Then there exists 
a sequence of smooth radial potentials $V_{n}: \mathbb{R}^{d} \rightarrow \mathbb{C}, n \in \mathbb{N}$, satisfying

$$
\left|V_{n}(x)\right| \lesssim(n+|x|)^{-1}
$$

and such that $\lambda$ is an eigenvalue of $T(D)+V_{n}(x)$ in $L^{2}\left(\mathbb{R}^{d}\right)$, for all $n \in \mathbb{N}$. In particular, $\lim _{n \rightarrow \infty}\left\|V_{n}\right\|_{L^{q}}=0$ for any $q>d$.

Theorem 1.4 (Chandrasekhar-Herbst operator). Let $H=\sqrt{-\Delta+1}-1$ be the Chandrasekhar-Herbst operator with mass 1 , and let $\lambda>0$. Then there exists a sequence of smooth potentials $V_{n}: \mathbb{R}^{d} \rightarrow \mathbb{R}, n \in \mathbb{N}$, satisfying

$$
\left|V_{n}(x)\right| \lesssim\left(n+\left|x^{\prime}\right|^{2}+\left|x_{d}\right|\right)^{-1}
$$

and such that $\lambda$ is an eigenvalue of $H+V_{n}$ in $L^{2}\left(\mathbb{R}^{d}\right)$, for every $n \in \mathbb{N}$.

Theorem 1.5 (Dirac operator). Let $\mathcal{D}_{m}=\alpha \cdot D+m \beta$ be the Dirac operator with mass $m \geq 0$ (see e.g. [26] for the definition of the Dirac matrices $\alpha_{j}, \beta \in \mathbb{C}^{K \times K}$ ) and let $\lambda \in(-\infty-m) \cup(m, \infty)$. There exists a sequence of smooth anti-hermitian potentials $V_{n}, n \in \mathbb{N}$, satisfying

$$
\left|V_{n}(x)\right| \lesssim\left(n+\left|x^{\prime}\right|^{2}+\left|x_{d}\right|\right)^{-1}
$$

and such that $\lambda$ is an eigenvalue of $\mathcal{D}+V_{n}$ in $L^{2}\left(\mathbb{R}^{d} ; \mathbb{C}^{K}\right)$, for every $n \in \mathbb{N}$.

Remark 1.6. All of the above theorems also hold when $\lambda$ is a critical value of $T$. In fact, this case is easier and somewhat less interesting since the distinction between the radial and the nonradial case disappears. An inspection of the proof of Theorem 1.1 will show that if $\lambda$ is a critical value, then one can even achieve the decay

$$
\left|V_{n}(x)\right| \lesssim(n+|x|)^{-2} .
$$

Remark 1.7. Using [5, Lemma 3.3] together with a Birman-Schwinger argument one can show that if $T: \mathbb{R}^{d} \rightarrow \mathbb{R}$ is smooth and proper (i.e. preimages of compact sets are compact), and if $M_{\lambda}$ has everywhere nonvanishing Gaussian curvature, then $\lambda$ cannot be an eigenvalue of $T(D)+V$, provided $\|V\|_{L^{(d+1) / 2}}+\|V\|_{L^{\infty}}$ is sufficiently small. We speculate that in the case of radial potentials a smallness assumption on $\|V\|_{L^{d}}+\|V\|_{L^{\infty}}$ would be sufficient. More precise results will appear elsewhere.

Remark 1.8. One can also show (see e.g. [6, Corollary A.9]) that if $M_{\lambda}$ has at least $k$ nonvanishing principal curvatures at every point, then $\lambda$ cannot be an eigenvalue, provided $\|V\|_{L^{(k+2) / 2}}+\|V\|_{L^{\infty}}$ is sufficiently small. Note that there is a gap between the $q$ in Theorem 1.2 and $q=(k+2) / 2$ here. The reason is that the latter does not consider worst-case scenarios. For instance, if $T(\xi)=\xi_{1}^{2}+\ldots+\xi_{k+1}^{2}$ where $k<d-1$ (i.e. $T$ depends on less than $d$ variables), then, e.g. for $\lambda=1$, the Fermi surface $M_{\lambda}$ is a cylinder, and the proof of Theorem 1.2 shows that one can get

$$
\left|V_{n}(x)\right| \lesssim\left(n+\left|x_{1}\right|^{2}+\ldots+\left|x_{k}\right|^{2}+\left|x_{k+1}\right|^{m}+\ldots+\left|x_{d-1}\right|^{m}+\left|x_{d}\right|\right)^{-1}
$$

for arbitrary positive $m$. Then $V_{n} \in L^{q}\left(\mathbb{R}^{d}\right)$ for all $q>(k+2) / 2+(d-1-k) / m$. This shows that the exponent $q=(k+2) / 2$ is in general optimal. 
Remark 1.9. By a transference-type argument one can prove analogues of Theorems $1.1-1.3$ to discrete Schrödinger operators on $\ell^{2}\left(\mathbb{Z}^{d}\right)$. In this case $T$ is a function on the torus $[0,2 \pi]^{d}$ and acts as (by slight abuse of notation)

$$
(T(D) f)(n):=(2 \pi)^{-d} \int_{[0,2 \pi]^{d}} \mathrm{e}^{\mathrm{i} x \cdot \xi} T(\xi) \widehat{f}(\xi) \mathrm{d} \xi, \quad n \in \mathbb{Z}^{d},
$$

on functions $f: \mathbb{Z}^{d} \rightarrow \mathbb{C}$ with rapid decay. Here,

$$
\widehat{f}(\xi):=\sum_{n \in \mathbb{Z}^{d}} \mathrm{e}^{-\mathrm{i} n \cdot \xi} f(n)
$$

is the discrete Fourier transform of $f$. An argument involving the Poisson summation formula shows that if $\left.f\right|_{\mathbb{Z}^{d}}$ is the restriction of a smooth function $f: \mathbb{R}^{d} \rightarrow \mathbb{C}$ with sufficient decay, then

$$
\left(\left.T(D) f\right|_{\mathbb{Z}^{d}}\right)(n)=\left(T_{\mathrm{per}}(D) f\right)(n), \quad n \in \mathbb{Z}^{d} .
$$

Here $T_{\text {per }}: \mathbb{R}^{d} \rightarrow \mathbb{C}$ is the periodization of $T:[0,2 \pi]^{d} \rightarrow \mathbb{C}$. On the left hand side $T(D)$ is understood as a multiplier on $\mathbb{Z}^{d}$ as in (16), while on the right hand side $T_{\text {per }}(D)$ is a multiplier on $\mathbb{R}^{d}$ in the sense of (10). Since Theorems 1.1-1.3 can be applied to $T_{\text {per }}$ and the eigenfunctions in the proofs may be chosen smooth and with arbitrary decay, the discrete analogues follow directly from (17).

Remark 1.10. An interesting example where the discrete analogue of Theorem 1.2 applies is the (standard) discrete Schrödinger operator

$$
H f(n):=-\frac{1}{4} \sum_{j=1}^{d}\left(f\left(n+e_{j}\right)-f\left(n-e_{j}\right)\right)+\frac{d}{2} f(n)+V(n) f(n)
$$

on $\ell^{2}\left(\mathbb{Z}^{d}\right)$. The symbol of the kinetic energy is

$$
T(\xi)=d-\sum_{j=1}^{d} \cos \left(\xi_{j}\right)
$$

and it is well known (see e.g. [13, Section 4.2]) that in $d \geq 3$ dimensions there are values of $\lambda$ such that the Fermi surface $M_{\lambda}$ has points of vanishing Gaussian curvature, i.e. $k<d-1$ in Theorem 1.2.

We briefly sketch the outline of this note. In Section 2 we prove Theorems 1.1 and Theorem 1.2 as well as some basic, but useful auxiliary results on mapping properties of pseudodifferential operators between spaces of functions with (anisotropic) symbol-type behavior. In Section 3 we connect the construction of the eigenfunctions used in the previous section to the Knapp example in harmonic analysis. In Section 4 we show that the potentials in Theorems 1.1-1.2 may be chosen real-valued under additional assumptions on the kinetic energy (see Proposition 4.2 and Corollary 4.3). In Section 5 we prove Theorems 1.3-1.5. 


\section{Proofs of Theorems $1.1-1.2$}

2.1. Anisotropic symbols. Let $\rho: \mathbb{R}^{d} \rightarrow[1, \infty)$ be a temperate weight, i.e. a continuous function with the property that there exists $s>0$ such that for every $x, y \in \mathbb{R}^{d}$

$$
\rho(x) \lesssim \rho(y)\langle x-y\rangle^{s} .
$$

Note that this implies in particular that

$$
\langle x\rangle^{-s} \lesssim \rho(x) \lesssim\langle x\rangle^{s} .
$$

We fix $\gamma=\left(\gamma_{1}, \ldots, \gamma_{d}\right) \in(0,1]^{d}$ such that

$$
\gamma_{d} \geq \gamma_{d-1} \geq \ldots \geq \gamma_{1} .
$$

Definition 2.1. Let $\ell \in \mathbb{R}$ and assume that $\rho, \gamma$ satisfy (18), (20). We say that $f \in S_{\gamma}^{\ell}\left(\mathbb{R}^{d}\right)$ if $f \in C^{\infty}\left(\mathbb{R}^{d}\right)$ and for any $\alpha \in \mathbb{N}_{0}^{d}$ there exist constants $C_{\alpha}>0$ such that for all $x \in \mathbb{R}^{d}$,

$$
\left|\partial^{\alpha} f(x)\right| \leq C_{\alpha} \rho(x)^{\ell} \rho(x)^{-\gamma_{1} \alpha_{1}} \ldots \rho(x)^{-\gamma_{d} \alpha_{d}} .
$$

We recall that $a \in C_{\mathrm{pol}}^{\infty}\left(\mathbb{R}^{d}\right)$, the space of smooth, polynomially bounded functions, if $a \in C^{\infty}\left(\mathbb{R}^{d}\right)$ and for any $\alpha \in \mathbb{N}_{0}^{d}$, there exist constants $C_{\alpha}>0, m_{\alpha} \in \mathbb{R}$ such that for all $x \in \mathbb{R}^{d}$,

$$
\left|\partial^{\alpha} a(\xi)\right| \leq C_{\alpha}\langle\xi\rangle^{m_{\alpha}} .
$$

Both $S_{\gamma}^{\ell}\left(\mathbb{R}^{d}\right)$ and $C_{\mathrm{pol}}^{\infty}\left(\mathbb{R}^{d}\right)$ are locally convex spaces when equipped with the seminorms

$$
\begin{aligned}
\|f\|_{n} & :=\sup _{|\alpha| \leq n} \sup _{x \in \mathbb{R}^{d}}\left|\rho(x)^{-\ell} \rho(x)^{\gamma_{1} \alpha_{1}} \ldots \rho(x)^{\gamma_{d} \alpha_{d}} f(x)\right|, \quad f \in S_{\gamma}^{\ell}\left(\mathbb{R}^{d}\right), \\
\|a\|_{n, m} & :=\sup _{|\alpha| \leq n} \sup _{x \in \mathbb{R}^{d}}\left|\langle\xi\rangle^{-m} \partial^{\alpha} a(\xi)\right|, \quad a \in C_{\mathrm{pol}}^{\infty}\left(\mathbb{R}^{d}\right),
\end{aligned}
$$

respectively. Let $h \in(0,1]$ and $\xi \in \mathbb{R}^{d}$. By abuse of notation we write

$$
h \xi:=\left(h^{\gamma_{1}} \xi_{1}, \ldots, h^{\gamma_{d}} \xi_{d}\right) \in \mathbb{R}^{d} .
$$

Here $h$ plays the role of a semiclassical parameter. Given $a \in C_{\mathrm{pol}}^{\infty}\left(\mathbb{R}^{d}\right)$ and $f \in S_{\gamma}^{\ell}\left(\mathbb{R}^{d}\right)$, we define

$$
(a(h D) f)(x):=(2 \pi)^{-d} \lim _{\epsilon \rightarrow 0} \iint \mathrm{e}^{\mathrm{i}(x-y) \cdot \xi} \varphi(\epsilon \xi) \varphi(\epsilon y) a(h \xi) f(y) \mathrm{d} y \mathrm{~d} \xi
$$

where $\varphi \in \mathcal{S}\left(\mathbb{R}^{d}\right)$ is fixed and satisfies $\varphi(0)=1$. Note that the integral in (24) is absolutely convergent, and an integration by parts argument (as in the proof of Proposition 2.2 below) shows that the definition is independent of the choice of $\varphi$. For $h=1$ the definition (24) coincides with the one of (10). We write it in this way to avoid the semiclassical Fourier transformation. We will set $h=1 / n$ later on. Alternatively, we could stick to $h=1$, but then we will have to consider functions depending on $n$ in the subsequent proofs. Both points of views are equivalent, and we freely switch between one and the other in later sections. 
Proposition 2.2. Let $a \in C_{\mathrm{pol}}^{\infty}\left(\mathbb{R}^{d}\right)$ Then

$$
a(h D): S_{\gamma}^{\ell}\left(\mathbb{R}^{d}\right) \rightarrow S_{\gamma}^{\ell}\left(\mathbb{R}^{d}\right)
$$

as a continuous map, with seminorm bounds independent of $h \in(0,1]$.

Proof. We prove that $g=a(h D) f$ satisfies (21) for $\alpha=0$. The proof of the general case is similar. For $f \in S_{\gamma}^{\ell}\left(\mathbb{R}^{d}\right)$ and $\epsilon \in(0,1]$ let

$$
g_{\epsilon}(x):=(2 \pi)^{-d} \iint \mathrm{e}^{\mathrm{i}(x-y) \cdot \xi} \varphi(\epsilon y) \varphi(\epsilon \xi) a(h \xi) f(y) \mathrm{d} y \mathrm{~d} \xi .
$$

Integration by parts yields

$$
g_{\epsilon}(x)=(2 \pi)^{-d} \iint \mathrm{e}^{\mathrm{i}(x-y) \cdot \xi} L_{1}^{l_{1}} L_{2}^{l_{2}} \varphi(\epsilon \xi) \varphi(\epsilon y) a(h \xi) f(y) \mathrm{d} y \mathrm{~d} \xi
$$

where $l_{1}, l_{2} \in \mathbb{N}$ and

$$
L_{1}=\frac{1+(x-y) \cdot D_{\xi}}{1+|x-y|^{2}}, \quad L_{2}=\frac{1-\xi \cdot D_{y}}{1+|\xi|^{2}} .
$$

Hence, there exists $m\left(l_{1}\right)>0$ such that

$$
\left|g_{\epsilon}(x)\right| \lesssim\|f\|_{l_{2}}\|a\|_{l_{1}, m\left(l_{1}\right)} \iint\langle x-y\rangle^{-l_{1}}\langle\xi\rangle^{-l_{2}+m\left(l_{1}\right)} \rho(y)^{\ell} \mathrm{d} y \mathrm{~d} \xi .
$$

By changing the roles of $x$ and $y$ in (18) we see that $\rho(y) \lesssim \rho(x)\langle x-y\rangle^{s}$. Choosing first $l_{1}$ so large that $-l_{1}+l s<-d$ and then $l_{2}$ so large that $-l_{2}+m\left(l_{1}\right)<-d$, we get

$$
\left|g_{\epsilon}(x)\right| \lesssim \rho(x)^{\ell}
$$

where the implicit constant is independent of $\epsilon$ and $h$. The claim follows by letting $\epsilon \rightarrow 0$.

Definition 2.3. For a smooth function $a: \mathbb{R}^{d} \rightarrow \mathbb{C}$ the Taylor support of $a$ at the origin is the set

$$
\mathcal{T}(a):=\left\{\alpha \in \mathbb{N}_{0}^{d}: \partial^{\alpha} a(0) \neq 0\right\} .
$$

The Newton polyhedron of $a$, denoted by $\mathcal{N}(a)$, is the convex hull of the set $\left\{\alpha+\mathbb{R}_{+}^{d}: \alpha \in \mathcal{T}(a)\right\}$. The set of vertices of $\mathcal{N}(a)$ is denoted by $\mathcal{V}(a)$ and is a subset of $\mathcal{T}(a)$.

Proposition 2.4. Let $a \in C_{\mathrm{pol}}^{\infty}\left(\mathbb{R}^{d}\right), f \in S_{\gamma}^{\ell}\left(\mathbb{R}^{d}\right)$ and assume that (20) holds. Moreover, assume that

$$
\emptyset \neq \mathcal{T}(a) \subset\left\{\alpha \in \mathbb{N}_{0}^{d}: \sum_{j=1}^{d} \gamma_{j} \alpha_{j} \geq 1\right\} .
$$

Then $a(h D) f \in h S_{\gamma}^{\ell-1}\left(\mathbb{R}^{d}\right)$.

Proof. Choose $k \in \mathbb{N}$ such that $k \gamma_{1} \geq 1$. By Taylor's theorem, we have

$$
a(\xi)=\sum_{|\alpha| \leq k} \frac{1}{\alpha !} \partial^{\alpha} a(0) \xi^{\alpha}+\sum_{|\alpha|=k} \xi^{\alpha} b_{\alpha}(\xi)
$$


where

$$
b_{\alpha}(\xi)=k \sum_{|\gamma|=k} \frac{\xi^{\gamma}}{\gamma !} \int_{0}^{1}(1-\theta)^{k-1} \partial^{\gamma} a(\theta \xi) \mathrm{d} \theta .
$$

Clearly $b_{\alpha} \in C^{\infty}\left(\mathbb{R}^{d}\right)$. By Proposition 2.2 , we thus have that $b_{\alpha}(h D)=\mathcal{O}_{S_{\gamma}^{\ell} \rightarrow S_{\gamma}^{\ell}}(1)$. By (20)-(21) and the choice of $k$, it follows that

$$
\left|\sum_{|\alpha|=k}(h D)^{\alpha} b_{\alpha}(h D) f(x)\right| \lesssim h \rho(x)^{\ell-1}
$$

Define

$$
\mathcal{V}_{k}(a):=\mathcal{V}(a) \cap\left\{\alpha \in \mathbb{N}_{0}^{d}:|\alpha| \leq k\right\} \subset \mathcal{T}(a)
$$

Then we can write

$$
\sum_{|\alpha| \leq k} \frac{1}{\alpha !} \partial^{\alpha} a(0) \xi^{\alpha}=\sum_{\alpha \in \mathcal{V}_{k}(a)} \xi^{\alpha} p_{\alpha}(\xi)
$$

where $p_{\alpha}$ are polynomials. In particular, $p_{\alpha}(h D)=\mathcal{O}_{S_{\gamma}^{\ell} \rightarrow S_{\gamma}^{\ell}}(1)$, so that $(21)$ and (26) imply

$$
\left|\sum_{\alpha \in \mathcal{V}_{k}(a)}(h D)^{\alpha} p_{\alpha}(h D) f(x)\right| \lesssim h \rho(x)^{\ell-1} .
$$

The claim is proved.

Proof of Theorem 1.1. Let $\eta \in M_{\lambda}$. Since $\lambda$ is a regular value, we have $\nabla T(\eta) \neq 0$. By rotating coordinates if necessary, we may assume that $\nabla T(\eta)=|\nabla T(\eta)| e_{d}$. We fix

$$
\rho(x):=\left(1+\left|x^{\prime}\right|^{4}+\left|x_{d}\right|^{2}\right)^{1 / 2}, \quad \gamma=(1 / 2, \ldots, 1 / 2,1)
$$

and define

$$
\psi(x):=\rho(x)^{-N}, \quad u(x):=\mathrm{e}^{\mathrm{i} \eta \cdot x} \psi(x),
$$

where $N>(d+1) / 4$. A change of variables shows that

$$
\int_{\mathbb{R}^{d}}|u(x)|^{2} \mathrm{~d} x=\left(\int_{\mathbb{R}^{d-1}}\left(1+\left|x^{\prime}\right|^{4}\right)^{-N} \mathrm{~d} x^{\prime}\right)\left(\int_{\mathbb{R}}\left(1+\left|x_{d}\right|^{2}\right)^{-N+\frac{d-1}{4}} \mathrm{~d} x_{d}\right)<\infty,
$$

i.e. $u \in L^{2}\left(\mathbb{R}^{d}\right)$. Proposition 2.2 then implies that $T(D) u \in L^{2}\left(\mathbb{R}^{d}\right)$ as well. Hence $u$ is in the domain of $T(D)$. Note that since

$$
T(D) \mathrm{e}^{\mathrm{i} \eta \cdot x}=\mathrm{e}^{\mathrm{i} \eta \cdot x} T(D+\eta)
$$

as operators on smooth functions with bounded derivatives, we may assume without loss of generality that $\eta=0$. For $n \geq 1$ let $h=1 / n$ and $u_{n}(x):=h^{N} u(h x)$, where $h x$ is defined as in (23). If $V_{n}(x)=W_{h}(h x)$, then

$$
\left(T(D)-\lambda+V_{n}\right) u_{n}=0 \Longleftrightarrow\left(T(h D)-\lambda+W_{h}\right) u=0 .
$$

Since $u$ has no zeros, we may write the second equation as $W_{h}=-(T(h D)-\lambda) u / u$. We will prove that

$$
\left|W_{h}(x)\right| \lesssim h\left(1+\left|x^{\prime}\right|^{2}+\left|x_{d}\right|\right)^{-1}
$$


This is equivalent to (11). Since (30) implies that $W_{h} \in L^{q}\left(\mathbb{R}^{d}\right)$ for $q>(d+1) / 2$, it also follows that

$$
\left\|V_{n}\right\|_{L^{q}\left(\mathbb{R}^{d}\right)}=h^{-\frac{d+1}{2 q}}\left\|W_{h}\right\|_{L^{q}\left(\mathbb{R}^{d}\right)}=\mathcal{O}\left(h^{1-\frac{d+1}{2 q}}\right) \rightarrow 0
$$

as $h \rightarrow 0$. To prove (30) we will apply Proposition 2.4 to $a(\xi)=T(\xi)-\lambda$ and $f=\psi$. It easy to check that $\psi \in S_{\gamma}^{-N}\left(\mathbb{R}^{d}\right)$ if $\gamma, \rho$ are defined as in (27). Moreover, condition (26) is clearly satisfied. Proposition 2.4 now yields that $W_{h} \in h S_{\gamma}^{-1}\left(\mathbb{R}^{d}\right)$; in particular, (30) holds.

Proof of Theorem 1.2. Let $\eta \in M_{\lambda}$ be such that only $k<d-1$ principal curvatures are nonzero at this point. We can again assume that $\eta$ is the origin and that $\nabla T(\eta)=|\nabla T(\eta)| e_{d}$. Write $x=\left(x^{\prime}, x^{\prime \prime}, x_{d}\right) \in \mathbb{R}^{k} \times \mathbb{R}^{d-1-k} \times \mathbb{R}$ and fix

$$
\rho(x):=\left(1+\left|x^{\prime}\right|^{4}+\left|x^{\prime \prime}\right|^{6}+\left|x_{d}\right|^{2}\right)^{1 / 2}, \quad \gamma=(\underbrace{1 / 2, \ldots, 1 / 2}_{k \text { times }}, \underbrace{1 / 3, \ldots, 1 / 3}_{d-1-k \text { times }}, 1) .
$$

We then define $u$ as in (28), but with $\rho$ as in (31). Near the origin, $M_{\lambda}$ is the graph of a smooth function $\Phi: \mathbb{R}^{k} \times \mathbb{R}^{d-1-k} \rightarrow \mathbb{R}$ over the last coordinate axis, and $\Phi(0)=0, \nabla \Phi(0)=0$. By the curvature assumption, we can write (after perhaps a linear change of the $\left(\xi^{\prime}, \xi^{\prime \prime}\right)$ coordinates)

$$
\Phi\left(\xi^{\prime}, \xi^{\prime \prime}\right)=\frac{1}{2} \sum_{j=1}^{k} s_{j} \xi_{j}^{2}+\mathcal{O}\left(\left|\left(\xi^{\prime}, \xi^{\prime \prime}\right)\right|^{3}\right) \quad\left(s_{j}= \pm 1\right),
$$

as $\left(\xi^{\prime}, \xi^{\prime \prime}\right) \rightarrow 0$, with similar estimates for all derivatives. By differentiating the identity $T\left(\xi^{\prime}, \xi^{\prime \prime}, \Phi\left(\xi^{\prime}, \xi^{\prime \prime}\right)\right)=\lambda$ twice, we find that

$$
\partial_{i} \partial_{j} T(0)=-\left(\partial_{d} T(0)\right)^{-1} \partial_{i} \partial_{j} \Phi(0), \quad i, j \leq d-1,
$$

and hence the Taylor support of $T(\xi)-\lambda$ at $\xi=0$ is contained in the set

$$
\left\{\alpha \in \mathbb{N}_{0}^{d}: \frac{1}{2}\left(\alpha_{1}+\ldots+\alpha_{k}\right)+\frac{1}{3}\left(\alpha_{k+1}+\ldots+\alpha_{d-1}\right)+\alpha_{d} \geq 1\right\} .
$$

We define $W_{h}:=-(T(h D)-\lambda) u / u$ and $V_{n}(x):=W_{h}(h x)$ for $h=1 / n$. As in the proof of Theorem 1.1 it is sufficient to prove that

$$
\left|W_{h}(x)\right| \lesssim h\left(1+\left|x^{\prime}\right|^{2}+\left|x^{\prime \prime}\right|^{3}+\left|x_{d}\right|\right)^{-1} .
$$

Indeed, (32) is equivalent to (12), and for any $q>(k+2) / 2+(d-1-k) / 3$, we have that

$$
\left\|V_{n}\right\|_{L^{q}\left(\mathbb{R}^{d}\right)}=h^{-\frac{1}{q}-\frac{k}{2 q}-\frac{d-k-1}{3 q}}\left\|W_{h}\right\|_{L^{q}\left(\mathbb{R}^{d}\right)}=\mathcal{O}\left(h^{1-\frac{1}{q}-\frac{k}{2 q}-\frac{d-k-1}{3 q}}\right) \rightarrow 0
$$

as $h \rightarrow 0$. The bound (32) follows from Proposition 2.4.

\section{Connection to Knapp's example}

Let us now quickly recall Knapp's homogeneity argument (see e.g. [10, Example 10.4.4]) for the sphere $S^{d-1}$. The same argument yields a necessary condition for the restriction problem of $M_{\lambda}$ if the latter has everywhere nonzero Gaussian 
curvature. For the present purpose it is more convenient to consider the restriction problem in its original (not adjoint) form

$$
\|\widehat{f}\|_{L^{q}\left(S^{d-1}\right)} \leq C\|f\|_{L^{p}\left(\mathbb{R}^{d}\right)}, \quad \text { for all } f \in \mathcal{S}\left(\mathbb{R}^{d}\right) .
$$

This is equivalent to (3). Since the problem is translation-invariant, we may assume that the north pole of the unit sphere is the origin of our coordinate system. Let $f=\chi_{R}$ be a smoothed version of the characteristic function for a rectangle $R$. Then for $0<\delta<1$ and $f_{\delta}(x):=f\left(\delta x^{\prime}, \delta^{2} x_{d}\right)$ one easily checks that

$$
\left\|f_{\delta}\right\|_{L^{p}\left(R^{d}\right)} \approx \delta^{-\frac{d+1}{p}}, \quad\left\|\widehat{f}_{\delta}\right\|_{L^{q}\left(S^{d-1}\right)} \approx \delta^{\frac{d-1}{q}-d-1} .
$$

Since $\delta<1$, this shows that (33) can only hold if the second condition in (4) is satisfied.

It is customary to call $f_{\delta}$ a Knapp example. If we look at its phase-space portrait we see that it is supported on a rectangle $R_{\delta}$ of size $\delta^{-1} \times \delta^{-2}$ and that its Fourier transform decays rapidly off the dual rectangle

$$
R_{\delta}^{*}:=\left\{\xi \in \mathbb{R}^{d}:|\xi|^{\prime} \lesssim \delta,\left|\xi_{d}\right| \lesssim \delta^{2}\right\} .
$$

Since

$$
T(\xi)=\lambda+\left(\partial_{d} T(0)\right) \xi_{d}+\mathcal{O}\left(|\xi|^{2}\right)
$$

as $|\xi| \rightarrow 0$, we thus have

$$
T(D) f_{\delta}(x)=\left.T\right|_{R_{\delta}^{*}} f_{\delta}(x)+\mathcal{O}\left(\delta^{2}\right)=\lambda f_{\delta}(x)+\mathcal{O}\left(\delta^{2}\right) .
$$

Since pseudodifferential operators do not move the support too much, this function is essentially supported (up to rapidly decaying tails) on the double of $R_{\delta}$. Cover $\mathbb{R}^{d} \backslash B(0,1)$ by finitely overlapping rectangles $R_{j}$ of size $2^{j} \times 4^{j}$ such that for $j \in \mathbb{N}$,

$$
x \in R_{j} \Longrightarrow\left|x^{\prime}\right|^{2}+\left|x_{d}\right| \approx 4^{j} .
$$

This can be done by a slight modification of the standard Littlewood-Paley decomposition. Let $\left\{\chi_{j}\right\}_{j=1}^{\infty}$ be a partition of unity subordinate to this cover and define

$$
u(x):=\sum_{j=1}^{\infty} 4^{-N j} \chi_{j}(x), \quad x \in \mathbb{R}^{d} \backslash B(0,1) .
$$

The function $u$ may be viewed as a superposition of Knapp examples $\left\{\chi_{j}\right\}_{j=1}^{\infty}$ corresponding to rectangles $\left\{R_{j}\right\}_{j=1}^{\infty}$. It is not difficult to show that, for $x$ in the exterior of the unit ball, we have that

$$
u(x) \approx\left(\left|x^{\prime}\right|^{2}+\left|x_{d}\right|\right)^{-N}
$$

as well as

$$
T(D) u(x)=\sum_{j=1}^{\infty} 4^{-N j}\left(\left.T\right|_{R_{j}^{*}}+\mathcal{O}\left(4^{-j}\right)\right) \chi_{j}(x)=\lambda u(x)+\mathcal{O}\left(\left(\left|x^{\prime}\right|^{2}+\left|x_{d}\right|\right)^{-N-1}\right) .
$$

Modifying $u$ in (34) by a compactly supported function, we easily arrange that $u>0$ and that $V=-(T(D)-\lambda) u / u$ is smooth and satisfies (11). The relation (35) connects the Knapp example and the Ionescu-Jerison example (i.e. the eigenfunction used in the proof of Theorem 1.1). There is a similar connection 
between a Knapp example for surfaces with $k$ non-vanishing principal curvatures and the eigenfunction used in the proof of Theorem 1.2.

\section{Real-Valued potentials}

Lemma 4.1. Let $T \in C_{\mathrm{pol}}^{\infty}\left(\mathbb{R}^{d}\right)$ be real-valued and time-reversal symmetric, i.e. $T(\xi)=T(-\xi)$. Let $\varphi(x)=\sin (\eta \cdot x) \psi(x)$ where $\psi \in C_{b}^{\infty}\left(\mathbb{R}^{d}\right)$ is any real-valued function and $\eta \in \mathbb{R}^{d}$. Then the function $T(D) \varphi$ is real-valued.

Proof. A straightforward calculation using (29) yields

$$
T(D) \varphi(x)=\frac{1}{2 \mathrm{i}}\left(\mathrm{e}^{\mathrm{i} \eta \cdot x} T(D+\eta)-\mathrm{e}^{-\mathrm{i} \eta \cdot x} T(D-\eta)\right) \psi(x) .
$$

Changing variables $\xi \rightarrow-\xi$ in the resulting integrals and using the fact that $\widehat{\psi}(\xi)=\widehat{\psi}(-\xi)$, one finds that $\overline{T(D) \varphi(x)}=T(D) \varphi(x)$.

Proposition 4.2. Assume that the assumptions of Theorem 1.1 or Theorem 1.2 hold and that $T$ is a polynomial such that $T(\xi)=T(-\xi)$ for all $\xi \in \mathbb{R}^{d}$. In addition, assume that, possibly after a linear bijection $L: \mathbb{R}^{d} \rightarrow \mathbb{R}^{d}$, we have $e_{1} \in M_{\lambda}$ and

$$
\mathcal{T}\left(T\left(e_{1}+\cdot\right) \cap\left\{\alpha \in \mathbb{N}_{0}^{d}:|\alpha| \in 2 \mathbb{N}-1 \text { and } \alpha_{1}=m\right\}=\left\{m e_{1}\right\}\right.
$$

for some odd integer $m$. Then the conclusion of Theorem 1.1 or Theorem 1.2 holds with real-valued potentials.

Proof. We first note that the assumptions on $T$ are invariant under linear bijections $L: \mathbb{R}^{d} \rightarrow \mathbb{R}^{d}$. Moreover, if $u$ is a real-valued function, then so is $u_{L}(x):=u\left({ }^{t} L x\right)$. Since

$$
\widehat{u_{L}}(\xi)=|\operatorname{det} L|^{-1} \widehat{u}\left(L^{-1} \xi\right),
$$

we have that $\left(T(D) u_{L}\right)(x)=\left(T_{L}(D) u\right)\left({ }^{t} L x\right)$, where $T_{L}(\xi)=T(L \xi)$. If $\eta \in$ $M_{\lambda} \backslash\{0\}$ we can arrange that $L e_{1}=\eta$. Since $T(\xi)=T(-\xi)$, the origin is a critical point, so there is no loss of generality in omitting it.

Assume now that the assumptions of the proposition hold with $L$ being the identity. We will prove the claim under the assumptions of Theorem 1.1. The case of Theorem 1.2 is analogous. We also assume that $n=1$ since the general case follows by scaling as in the proof of Theorem 1.1.

We would like to choose the eigenfunction as in Lemma 4.1, i.e.

$$
\widetilde{u}(x):=\sin \left(x_{1}\right) \psi(x)
$$

where $\psi$ is given by

$$
\psi(x):=\left(1+\left|P_{\nu} x\right|^{2}+\left|P_{\nu}^{\perp} x\right|^{4}\right)^{-N / 2}
$$

with $N>d / 2$. Here $P_{\nu}$ is the orthogonal projection onto the subspace spanned by the unit vector $\nu=\nabla T\left(e_{1}\right) /\left|\nabla T\left(e_{1}\right)\right|$ and $P_{\nu}^{\perp}=\mathrm{id}-\mathrm{P}_{\nu}$. The problem is that $\widehat{u}$ now vanishes on the hypersurfaces $x_{1}=k \pi, k \in \mathbb{Z}$, so $-(T(D)-\lambda) \widetilde{u} / \widetilde{u}$ may be singular. We follow the basic strategy of Ionescu and Jerison [11] to remove the singularities. We are looking for a function $u$ with the same zero set (including multiplicities) as $\widetilde{u}$ such that $(T(D)-\lambda) u=0$ on this set. We first compute

$$
T(D) \widetilde{u}(x)=\frac{1}{2 \mathrm{i}} \mathrm{e}^{\mathrm{i} x_{1}}\left(T\left(D+e_{1}\right)-T\left(D-e_{1}\right)\right) \psi(x)+\sin \left(x_{1}\right) T\left(D-e_{1}\right) \psi(x) .
$$


Set $f=(T(D)-\lambda) \widetilde{u}$. As in the proof of Theorem 1.1 we use Proposition 2.4 to infer that

$$
\left|\partial^{\alpha} f(x)\right| \leq C\left(1+\left|P_{\nu} x\right|+\left|P_{\nu}^{\perp} x\right|^{2}\right)^{-1} \psi(x), \quad|\alpha| \leq m .
$$

In fact, $a(\xi)=T\left(\xi+e_{1}\right)-T\left(\xi-e_{1}\right)$ as well as $a(\xi)=T\left(\xi-e_{1}\right)-\lambda$ satisfy the assumptions of Proposition 2.4 (after relabeling coordinates). For example, in the first instance, $a(0)=T\left(e_{1}\right)-T\left(-e_{1}\right)=0$ and $\nabla a(0)=\nabla T\left(e_{1}\right)-\nabla T\left(-e_{1}\right)=$ $2 \nabla T\left(e_{1}\right)$. Here we used the time-reversal symmetry $T(\xi)=T(-\xi)$ and $\nabla T(\xi)=$ $-\nabla T(-\xi)$. Define

$$
w(x):=\sum_{k \in \mathbb{Z}} \cos (k \pi)\left(x_{1}-k \pi\right)^{m} \chi\left(x_{1}-k \pi\right) f\left(k \pi, x^{\prime}\right)
$$

where $x^{\prime}=\left(x_{2}, \ldots, x_{d}\right) \in \mathbb{R}^{d-1}$ and $\chi \in C_{c}^{\infty}(\mathbb{R},[0,1])$ is supported on $[-c, c]$ and equals 1 on $[-c / 2, c / 2]$ for some small $c>0$ to be determined later. We then set

$$
u(x):=\sin \left(x_{1}\right)(\psi(x)-\kappa w(x)), \quad \kappa:=\left[(-1)^{\frac{m+1}{2}} \partial_{1}^{m} T\left(e_{1}\right)\right]^{-1} .
$$

By (38) we have

$$
|u(x)| \geq\left|\sin \left(x_{1}\right)\right| \psi(x)\left(1-C \kappa c^{m}\right) \gtrsim\left|\sin \left(x_{1}\right)\right| \psi(x) \gtrsim \operatorname{dist}\left(x_{1}, \pi \mathbb{Z}\right) \psi(x)
$$

if $c$ is chosen sufficiently small. We now fix $c<\pi / 10$ such that (41) holds. This also guarantees that the functions $\chi(\cdot-k \pi), k \in \mathbb{Z}$ have mutually disjoint supports. A straightforward computation yields

$$
\begin{aligned}
(T(D)-\lambda) u(x) & =f(x)-\frac{\kappa}{2 \mathrm{i}} \sum_{|\alpha| \geq 1} \frac{1}{\alpha !}\left(e^{\mathrm{i} x_{1}}-(-1)^{|\alpha|} e^{-\mathrm{i} x_{1}}\right) \partial^{\alpha} T\left(e_{1}\right) D^{\alpha} w(x) \\
& =f(x)-\kappa \sin \left(x_{1}\right) \sum_{\alpha \in 2 \mathbb{N}} \frac{1}{\alpha !} \partial^{\alpha} T\left(e_{1}\right)(-1)^{\frac{|\alpha|}{2}} \partial^{\alpha} w(x) \\
& -\kappa \cos \left(x_{1}\right) \sum_{\alpha \in 2 \mathbb{N}-1} \frac{1}{\alpha !} \partial^{\alpha} T\left(e_{1}\right)(-1)^{\frac{|\alpha|+1}{2}} \partial^{\alpha} w(x) .
\end{aligned}
$$

This together with (38), (39) implies that

$$
|(T(D)-\lambda) u(x)| \lesssim\left(1+\left|P_{\nu} x\right|+\left|P_{\nu}^{\perp} x\right|^{2}\right)^{-1} \psi(x) .
$$

Using (36) and (42) we check that, for $k \in \mathbb{Z}$,

$$
\begin{aligned}
& (T(D)-\lambda) u\left(k \pi, x^{\prime}\right)=f\left(k \pi, x^{\prime}\right) \\
& -\left.\kappa \sum_{|\alpha| \in 2 \mathbb{N}-1} \frac{1}{\alpha !} \partial^{\alpha} T\left(e_{1}\right)(-1)^{\frac{|\alpha|+1}{2}} \partial^{\alpha}\left(\left(x_{1}-k \pi\right)^{m} \chi\left(x_{1}-k \pi\right) f\left(k \pi, x^{\prime}\right)\right)\right|_{x_{1}=k \pi} \\
& =f\left(k \pi, x^{\prime}\right)\left(1-\kappa(-1)^{\frac{m+1}{2}} \partial_{1}^{m} T\left(e_{1}\right)\right)=0 .
\end{aligned}
$$

To see this, split the sum into a part where $\alpha=m e_{1}$ and its complement. The first part gives exactly the expression in the last line. The complementary part can be further split into three subparts where $\alpha_{1}<m, \alpha_{1}>m$ or $\alpha_{1}=m$. The first subpart is zero because there are too few $x_{1}$-derivatives that can fall onto the monomial $\left(x_{1}-k \pi\right)^{m}$. The second subpart is zero because there is at least one 
$x_{1}$-derivative that must fall onto the cutoff function $\chi$, and this is constant near $x_{1}=k \pi$. The third subpart is zero by assumption (36).

Combining the result of the previous computation with (43) we get

$$
|(T(D)-\lambda) u(x)| \lesssim\left(1+\left|P_{\nu} x\right|+\left|P_{\nu}^{\perp} x\right|^{2}\right)^{-1} \operatorname{dist}\left(x_{1}, \pi \mathbb{Z}\right) \psi(x) .
$$

This together with (41) yields that

$$
|V(x)|=\frac{|(T(D)-\lambda) u(x)|}{|u(x)|} \lesssim\left(1+\left|P_{\nu} x\right|+\left|P_{\nu}^{\perp} x\right|^{2}\right)^{-1} .
$$

That $V$ is real-valued follows from Lemma 4.1.

Corollary 4.3. Assume that the assumptions of Theorem 1.1 or Theorem 1.2 hold and that $T$ is a radial polynomial. Then the conclusion of Theorem 1.1 or Theorem 1.2 holds with real-valued potentials.

Proof. The assumptions imply that $T$ is of the form $T(\xi)=T_{0}(|\xi|)$ where

$$
T_{0}(r)=\sum_{j=0}^{K} c_{j} r^{2 j}
$$

for some $K \in \mathbb{N}$ and $\left(c_{j}\right)_{j=1}^{K} \subset \mathbb{R}$. If $\eta \in M_{\lambda}$, then $|\eta| e_{1} \in M_{\lambda}$. The linear bijection $L \xi=|\eta| \xi$ preserves spherical symmetry, and $T_{L}\left(e_{1}\right)=T\left(L e_{1}\right)=\lambda$. Assuming, as we may, that $K \neq 0$, we have

$$
\left|\xi+e_{1}\right|^{2 K}=\left(1+2 \xi_{1}+\xi_{1}^{2}+\ldots+x_{d}^{2}\right)^{K}=\xi_{1}^{2 K}+2 K \xi_{1}^{2 K-1}+Q_{K}(\xi)
$$

where $Q_{K}$ is a sum of monomials that do not contain the factor $\xi_{1}^{2 K-1}$. Similarly, none of the monomials appearing in $\left|\xi+e_{1}\right|^{2 j}, j \leq K-1$, contain this factor. Therefore, condition (36) holds with $m=2 K-1$.

\section{Proofs of Theorems $1.3-1.5$}

We first state a generalization of Propositions 2.2 and 2.4 to symbols $a(x, \xi)$ depending on $x$. We restrict ourselves to the case

$$
\rho(x)=\left(1+|x|^{2}\right)^{1 / 2}, \quad \gamma=(1, \ldots, 1) ;
$$

more general cases will not be needed. The proof is a straightforward adaptation of that of Propositions 2.2 and 2.4 and will be omitted.

Lemma 5.1. Assume that $a \in C^{\infty}\left(\mathbb{R}^{d} \times \mathbb{R}^{d}\right)$ and that

$$
\left|\partial_{x}^{\alpha} \partial_{\xi}^{\beta} a(x, \xi)\right| \leq C_{\alpha, \beta}(1+|x|)^{k-|\alpha|}(1+|\xi|)^{m_{\alpha, \beta}} .
$$

Then $a(x, h D): S_{\gamma}^{\ell}\left(\mathbb{R}^{d}\right) \rightarrow S_{\gamma}^{\ell+k}\left(\mathbb{R}^{d}\right)$ as a continuous map, with seminorms bounded independent of $h \in(0,1]$. Moreover, if $a(x, 0)=0$ for all $x \in \mathbb{R}^{d}$, then $a(x, h D): S_{\gamma}^{\ell}\left(\mathbb{R}^{d}\right) \rightarrow h S_{\gamma}^{\ell+k-1}\left(\mathbb{R}^{d}\right)$.

Proof of Theorem 1.3. We will prove the claim for $n=1$; the general case follows from a scaling argument similar to that in the proofs of Theorems 1.1-1.2.

Since $T$ is a radial, nonconstant polynomial, the Fermi surface $M_{\lambda}$ is a finite union of spheres. Let $\sigma_{\lambda}$ be the uniform surface measure of one of these spheres. Clearly, $\sigma_{\lambda}^{\vee}$ is radial. We fix $r_{0} \gg 1$ and pick a smooth radial function $\varphi$ that 
is positive for $|x| \leq r_{0}$ and equal to $\sigma_{\lambda}^{\vee}$ for $|x|>r_{0}$. We then define the radial function

$$
\widetilde{u}(x):=\varphi(x) \psi(x),
$$

where $\psi(x):=\left(1+|x|^{2}\right)^{-N / 2}$, with $N>d / 2$. A straightforward calculation using Fubini and the convolution theorem yields that, for $|x|>r_{0}$,

$$
f(x):=(T(D)-\lambda) \widetilde{u}(x)=a(x, D) \psi(x)
$$

where

$$
a(x, \xi)=(2 \pi)^{-d} \int_{M_{\lambda}} \mathrm{e}^{\mathrm{i} \eta \cdot x}(T(\xi+\eta)-\lambda) \mathrm{d} \sigma_{\lambda}(\eta) .
$$

Since $M_{\lambda}$ is compact, it follows from stationary phase (see e.g. [23, (1.2.8)]) that

$$
\left|\partial_{x}^{\alpha} \partial_{\xi}^{\beta} a(x, \xi)\right| \leq C_{\alpha, \beta}(1+|x|)^{-\frac{d-1}{2}-|\alpha|}(1+|\xi|)^{m_{\alpha, \beta}} .
$$

Since $a(x, 0)=0$, Lemma 5.1 yields

$$
|f(x)| \lesssim(1+|x|)^{-\frac{d-1}{2}-1} \psi(x) .
$$

After rescaling, we may assume that $M_{\lambda}$ is the unit sphere $S^{d-1}$. From (6) it follows that that the zeros of $\widetilde{u}$ are simple and the distance between consecutive zeros is uniformly bounded below by a constant $\delta>0$. Let us denote these zeros by $Z:=\left\{r_{k}\right\}_{k=1}^{\infty} \subset\left(r_{0}, \infty\right)$. Then we have that

$$
|\widetilde{u}(x)| \gtrsim \operatorname{dist}(|x|, Z)(1+|x|)^{-\frac{d-1}{2}} \psi(x) .
$$

Pick $\chi \in C_{c}^{\infty}(\mathbb{R},[0,1])$ as in the proof of Proposition 4.2, i.e. $\chi$ is supported on $[-c, c]$ and equals 1 on $[-c / 2, c / 2]$ for some sufficiently small $c>0$; in particular, we require $c<\delta / 4$, so that the functions $\chi\left(\cdot-r_{k}\right)$ have mutually disjoint support. By assumption, $T(D)$ is a differential operator of the form

$$
T(D)=\sum_{j=0}^{m} c_{j}(-\Delta)^{j}
$$

where $\left\{c_{j}\right\}_{j=0}^{m} \subset \mathbb{R}, c_{m} \neq 0$ and $m \geq 1$. Define the radial functions

$$
w(x):=\sum_{k=1}^{\infty}\left(|x|-r_{k}\right)^{2 m} \chi\left(|x|-r_{k}\right) f\left(r_{k}\right),
$$

and

$$
u(x):=\widetilde{u}(x)-\kappa w(x), \quad \kappa:=c_{m}^{-1}(2 d)^{-m} .
$$

It follows from (46) and (47) that

$$
|u(x)| \gtrsim \operatorname{dist}(|x|, Z) \psi(x)(1+|x|)^{-\frac{d-1}{2}}\left(1-\mathcal{O}\left(c^{2 m-1}\right)\right) .
$$

Moreover, for $|x|=r_{k}$ we have

$$
(T(D)-\lambda) u(x)=f_{0}\left(r_{k}\right)-\kappa c_{m}(2 d)^{m} f_{0}\left(r_{k}\right)=0,
$$

where we used the notation $f(x)=f_{0}(|x|)$. This together with (46) yields

$$
|(T(D)-\lambda) u(x)| \lesssim \operatorname{dist}(|x|, Z) \psi(x)(1+|x|)^{-\frac{d-1}{2}-1} .
$$


Hence, for $c$ suffiently small, we have by (48)-(49)

$$
|V(x)|=\frac{|(T(D)-\lambda) u(x)|}{|u(x)|} \lesssim(1+|x|)^{-1} .
$$

It is clear that $V$ is radial.

Proof of Theorem 1.4. Here we combine our previous results with ideas from [19] and [9]. Observe that if $\kappa:=\sqrt{(\lambda+1)^{2}-1}$, then $\kappa e_{d} \in M_{\lambda}$. As before, we may assume (after a rescaling) that $\kappa=1$ (and hence $\lambda=\sqrt{2}-1$ ). First define

$$
\begin{aligned}
g(t) & :=\int_{0}^{t} \sin ^{2}(y) \mathrm{d} y, \quad t \geq 0, \\
w_{n}(x) & :=\left(n^{2}+\left|x^{\prime}\right|^{4}+g\left(x_{d}\right)^{2}\right)^{-N / 2},
\end{aligned}
$$

where $n \in \mathbb{N}, N>(d+1) / 4$, and $g$ is extended to an even function on $\mathbb{R}$ in the definition of $w_{n}$. We then set

$$
\begin{aligned}
& \phi_{n}(x):=\left(\sqrt{\left(D+e_{d}\right)^{2}+1}+\sqrt{\left(D-e_{d}\right)^{2}+1}\right) w_{n}(x), \\
& u_{n}(x):=\sin \left(x_{d}\right) \phi_{n}(x) .
\end{aligned}
$$

The eigenvalue equation will hold if we set $V_{n}=-(T(D)-\lambda) u_{n} / u_{n}$, as usual. We have to prove that this is a smooth function decaying as in (11). An elementary computation (compare (37)) yields

$$
V_{n}(x)=\mathrm{e}^{\mathrm{i} x_{1}} \frac{\partial_{x_{d}} w_{n}(x)}{\sin \left(x_{d}\right) \phi_{n}(x)}-\frac{\left(T\left(D-e_{d}\right)-\lambda\right) \phi_{n}(x)}{\phi_{n}(x)} .
$$

This is fortunate since the term with the sine in the denominator now has a differential (i.e. local) operator in the nominator (similar to the situation in Proposition 4.2). The zeros of the denominator will be canceled by the choice of $w_{n}$. In fact, using that $1+g\left(x_{d}\right) \gtrsim\left|x_{d}\right|$, we have

$$
\left|\frac{\partial_{x_{d}} w_{n}(x)}{\sin \left(x_{d}\right)}\right| \lesssim\left(n+\left|x^{\prime}\right|^{2}+\left|x_{d}\right|\right)^{-N / 2-1}
$$

for large enough $n$. Next, we would like to apply Proposition 2.4 to the denominator in the second term of $V_{n}$ with

$$
a(\xi):=\left(T\left(\xi-e_{d}\right)-\lambda\right)\left(\sqrt{\left(\xi+e_{d}\right)^{2}+1}+\sqrt{\left(\xi-e_{d}\right)^{2}+1}\right)
$$

and $f=w_{n}$. Notice that $a(0)=0$ by the assumption that $e_{1} \in M_{\lambda}$. Here we cannot use the full strength of Proposition 2.4 since $w_{n}$ is not a symbol. It only satisfies the weaker estimates

$$
\begin{aligned}
\left|w_{n}(x)\right| & \lesssim\left(n+\left|x^{\prime}\right|^{2}+\left|x_{d}\right|\right)^{-N / 2} \\
\left|\partial^{\alpha} w_{n}(x)\right| & \lesssim\left(n+\left|x^{\prime}\right|^{2}+\left|x_{d}\right|\right)^{-N / 2-1}, \quad|\alpha| \geq 1 .
\end{aligned}
$$

However, as is obvious from the proof of Proposition 2.4, this still implies that

$$
\left|a(D) w_{n}(x)\right| \lesssim\left(n+\left|x^{\prime}\right|^{2}+\left|x_{d}\right|\right)^{-N / 2-1}
$$

It remains to prove that

$$
\left|\phi_{n}(x)\right| \gtrsim\left(n+\left|x^{\prime}\right|^{2}+\left|x_{d}\right|\right)^{-N / 2}
$$


since (50)-(53) then imply the desired properties of $V_{n}$. We write

$$
\begin{aligned}
\phi_{n}(x) & =2 \sqrt{2} w_{n}(x)+a(D) w_{n}(x), \\
a(\xi) & :=\sqrt{\left(\xi+e_{d}\right)^{2}+1}+\sqrt{\left(\xi-e_{d}\right)^{2}+1}-2 \sqrt{2} .
\end{aligned}
$$

Since $a(0)=0$, the same modification of Proposition 2.4 as above yields

$$
\begin{aligned}
\left|\phi_{n}(x)\right| & \geq 2 \sqrt{2} w_{n}(x)-\mathcal{O}(1)\left(n+\left|x^{\prime}\right|^{2}+\left|x_{d}\right|\right)^{-N / 2-1} \\
& \gtrsim\left(n+\left|x^{\prime}\right|^{2}+\left|x_{d}\right|\right)^{-N / 2}(1-\mathcal{O}(1 / n))
\end{aligned}
$$

Hence (53) will be satisfied for $n$ sufficiently large.

Proof of Theorem 1.5. Assume first that $m=0$. By scaling, we may then assume that $\lambda= \pm 1$. We fix an eigenvector $\mathbf{v} \in \mathbb{C}^{K}$ corresponding to the eigenvalue \pm 1 of the matrix $\alpha_{d}$ and define

$$
\begin{aligned}
& u_{n}(x):=\mathrm{e}^{\mathrm{i} x_{d}} \psi_{n}(x) \mathbf{v}, \\
& \psi_{n}(x):=\left(n^{2}+\left|x^{\prime}\right|^{4}+\left|x_{d}\right|^{2}\right)^{-N / 2}
\end{aligned}
$$

with $n \in \mathbb{N}$ and $N>(d+1) / 4$. We then compute

$$
\left(\mathcal{D}_{0}-\lambda\right) u(x)=\mathrm{e}^{\mathrm{i} x_{d}} \sum_{j=1}^{d} D_{j} \psi_{n}(x) \alpha_{j} \mathbf{v} .
$$

If we set

$$
V_{n}(x):=-\frac{1}{\psi_{n}(x)} \sum_{j=1}^{d} D_{j} \psi_{n}(x) \alpha_{j}
$$

then we obtain

$$
\left(\mathcal{D}_{0}+V_{n}(x)-\lambda\right) u_{n}(x)=0 .
$$

Since $\alpha_{j}$ are hermitian matrices, $\psi_{n}$ is real-valued and $D_{j}=-\mathrm{i} \partial_{j}$, it follows that $V_{n}$ is anti-hermitian. The proof for $m>0$ is similar; here one observes that the eigenvalues of the matrix $\alpha_{d}+m \beta$ are given by $\pm \sqrt{1+m^{2}}$.

\section{REFERENCES}

[1] S. Agmon. Lower bounds for solutions of Schrödinger equations. J. Analyse Math., 23:1-25, 1970.

[2] A. Berthier and V. Georgescu. On the point spectrum of Dirac operators. J. Funct. Anal., 71(2):309-338, 1987.

[3] N. Boussaï d and A. Comech. On spectral stability of the nonlinear Dirac equation. J. Funct. Anal., 271(6):1462-1524, 2016.

[4] A. Carey, F. Gesztesy, J. Kaad, G. Levitina, R. Nichols, D. Potapov, and F. Sukochev. On the global limiting absorption principle for massless Dirac operators. Ann. Henri Poincaré, 19(7):1993-2019, 2018.

[5] J.-C. Cuenin. Eigenvalue bounds for Dirac and fractional Schrödinger operators with complex potentials. J. Funct. Anal., 272(7):2987-3018, 2017.

[6] J.-C. Cuenin and P. Siegl. Eigenvalues of one-dimensional non-self-adjoint dirac operators and applications. Letters in Mathematical Physics, pages 1-22, Jan 2018.

[7] M. S. P. Eastham and H. Kalf. Schrödinger-type operators with continuous spectra, volume 65 of Research Notes in Mathematics. Pitman (Advanced Publishing Program), Boston, Mass.-London, 1982. 
[8] R. L. Frank. Eigenvalue bounds for Schrödinger operators with complex potentials. Bull. Lond. Math. Soc., 43(4):745-750, 2011.

[9] R. L. Frank and B. Simon. Eigenvalue bounds for Schrödinger operators with complex potentials. II. J. Spectr. Theory, 7(3):633-658, 2017.

[10] L. Grafakos. Modern Fourier analysis, volume 250 of Graduate Texts in Mathematics. Springer, New York, third edition, 2014.

[11] A. D. Ionescu and D. Jerison. On the absence of positive eigenvalues of Schrödinger operators with rough potentials. Geom. Funct. Anal., 13(5):1029-1081, 2003.

[12] A. D. Ionescu and W. Schlag. Agmon-Kato-Kuroda theorems for a large class of perturbations. Duke Math. J., 131(3):397-440, 2006.

[13] H. Isozaki and H. Morioka. Inverse scattering at a fixed energy for discrete Schrödinger operators on the square lattice. Ann. Inst. Fourier (Grenoble), 65(3):1153-1200, 2015.

[14] H. Kalf. Nonexistence of eigenvalues of Dirac operators. Proc. Roy. Soc. Edinburgh Sect. A, 89(3-4):309-317, 1981.

[15] T. Kato. Growth properties of solutions of the reduced wave equation with a variable coefficient. Comm. Pure Appl. Math., 12:403-425, 1959.

[16] C. E. Kenig and N. Nadirashvili. A counterexample in unique continuation. Math. Res. Lett., 7(5-6):625-630, 2000.

[17] H. Koch and D. Tataru. Sharp counterexamples in unique continuation for second order elliptic equations. J. Reine Angew. Math., 542:133-146, 2002.

[18] H. Koch and D. Tataru. Carleman estimates and absence of embedded eigenvalues. Comm. Math. Phys., 267(2):419-449, 2006.

[19] J. Lorinczi and I. Sasaki. Embedded eigenvalues and Neumann-Wigner potentials for relativistic Schrödinger operators. J. Funct. Anal., 273(4):1548-1575, 2017.

[20] C. Muscalu and W. Schlag. Classical and multilinear harmonic analysis. Vol. I, volume 137 of Cambridge Studies in Advanced Mathematics. Cambridge University Press, Cambridge, 2013.

[21] M. Reed and B. Simon. Methods of modern mathematical physics. IV. Analysis of operators. Academic Press [Harcourt Brace Jovanovich, Publishers], New York-London, 1978.

[22] B. Simon. On positive eigenvalues of one-body Schrödinger operators. Comm. Pure Appl. Math., 22:531-538, 1969.

[23] C. D. Sogge. Fourier integrals in classical analysis, volume 105 of Cambridge Tracts in Mathematics. Cambridge University Press, Cambridge, 1993.

[24] E. M. Stein. Harmonic analysis: real-variable methods, orthogonality, and oscillatory integrals, volume 43 of Princeton Mathematical Series. Princeton University Press, Princeton, NJ, 1993. With the assistance of Timothy S. Murphy, Monographs in Harmonic Analysis, III.

[25] E. M. Stein and R. Shakarchi. Functional analysis, volume 4 of Princeton Lectures in Analysis. Princeton University Press, Princeton, NJ, 2011. Introduction to further topics in analysis.

[26] B. Thaller. The Dirac equation. Texts and Monographs in Physics. Springer-Verlag, Berlin, 1992.

[27] V. Vogelsang. Absence of embedded eigenvalues of the Dirac equation for long range potentials. Analysis, 7(3-4):259-274, 1987.

[28] E. P. Wigner and J. Von-Neumann. Über merkwürdige Eigenwerte. Z. Phys, 30:465, 1929.

Mathematisches Institut, Ludwig-Maximilians-Universität München, 80333 Munich, GERMANY

E-mail address: cuenin@math.lmu.de 